\title{
Dipterologische Miscellen.
}

\author{
(2. Serie.)
}

Von Prof. Josef Mik in Wien.

VII.*)

43. Zur Biologie von Geron gibbosus Meig. - Herr O. Werner, derzeit am Landesmuseum in Sarajevo (Bosnien), hat Anfangs Juni 1891 bei Cuciste auf der Insel Sabioncello (Dalmatien) Raupen von Nephopteryx sublineatella Strg. (Pyralidina) und von Fumea crassiorella Brd. (Psychidae) gesammelt und nach Wien gebracht. Hier entwickelten sich bei der Aufzucht dieser Raupen im Juli desselben Jahres 3 Männchen einer Fliege, welche ich als Geron gibbosus Meig. bestimmte, und zwar eines aus Nephopteryx, die zwei anderen aus Fumea. Alle drei Exemplare befinden sich in meiner Sammlung. Aus dem Gesagten folgt, dass die Raupen der genannten Lepidopteren-Arten als Wirthe des Bombyliers, über dessen Lebensweise meines Wissens bisher nichts bekannt war, anzusehen sind. Es ist anzunehmen, dass Geron gibbosus auch noch in den Raupen anderer kleinerer Lepidopteren schmarotzen dürfte.

44. Herr Th. Becker hat in der Berl. Entom. Ztschrft. XXXI. 1887, pag. 135 eine Tachydromia testacea n. sp. beschrieben. Nachdem Philippi im Jahre 1865 (in den Verhandl. der k. k. Zoolog:-Botan. Gesellsch. zu Wien, pag. 767) einen Platypalpus testaceus aus Chile publicirt hat und das Genus Platypalpus mit dem Genus Tachydromia zusammenfällt, somit derselbe Artname zweimal in Verwendung käme, ändere ich den Namen Tachydr. testacea Beck. in Tachydromia engadinica m. (nom. nov.) um. - Auch Rhamphomyia angustipennis Beck. (Berl. Entom. Ztschrft. 1887, pag. 117) ist ein vergebener Name, indem Lo ew schon im Jahre 1861 (Berl. Ent. Ztschrft. pag. 336) eine Rhamph. angustipennis aus New-York beschrieben hat. Da aber Rh. angustipennis Beck. mit Rh. stigmosa Macq. zusammenfällt (conf. Strobl: Die Dipteren von Steiermark, 1893, pag. 50), so ist eine nene Namengebung nicht nothwendig.

*) VI.: Siehe Wien. Entom. Ztg. 1895, pag. 93. 
45. Herr Prof. Thom as hat im Programm der Herzogl. Realschule und des Progymnasiums zu Ohrdruf 1885, pag. 4 eine Hypertrophie der Fruchtknoten von Salix reticulata L. durch eine Cecidomyiden-Larve bekannt gemacht und dieselbe auch später (im Programm des Gymnasium Gleichense zu Ohrdruf 1892, pag. 15, Nr. 23) von einem anderen Standorte angezeigt, bei welcher Gelegenheit er schreibt: „dieses Cecidium ist von anderen Standorten meines Wissens bisher nicht bekannt geworden." Ich bemerke hiezu, dass Fr. Loew diese Deformation auch aus dem Gschnitzthale in Tirol erhalten hat (conf. Verhandl. der k. k. Zoolog.-Botan. Gesellsch. Wien, 1888, pg. 244).

46. Ich halte Helomyza tuberiperda Rond., Atti della Soc. Ital. di Scienze Naturali, Vol. X. Milano 1867, pag. 122, Nr. 10, für Helomyza hispanica Lw., Ztschrft. für Entomologie im Auftrage des Vereins für schlesische Insectenkunde, Breslau 1859, pag. 27, Nr. 8; wenigstens spricht bei Vergleichung der Beschreibungen beider Arten nichts gegen meine Auffassung.

47. Herr Abbé K i effer schreibt in den Ent. Nachricht. (Jahrg. 1894, pag. 296) über eine Galle auf Centaurea Scabiosa L. Folgendes: "Aulax sp.? Fleischige, schlehen- bis walnussdicke, vielkammerige, unterirdische Auswüchse am Stengelgrunde" und ein paar Zeilen weiter: „Eine ähnliche Galle wird von Schiner (Fauna Austr. Diptera) der Trypeta eluta Meig. zugeschrieben, und zwar nach einer Beobachtung von Kaltenbach. Da aber Kaltenbach in seinem 1874, also 10 Jahre später veröffentlichten Werke - Die Pflanzenfeinde - von dieser Fliege angibt, dass sie in den Blüthenköpfen von Centaurea Scabiosa vorkomme, so wird dadurch die Richtigkeit der Schinerschen Angabe in Frage gestellt." - Es liegt hier von Seiten Herrn Kieffer's ein Irrthum vor; denn Schiner bezieht sich in seinem Citate über diese von der erwähnten Bohrfliege verursachten Stengelgallen weder aut $\mathrm{K}$ altenbach, noch auf eine Centaurea. Zur Richtigstellung der Sache empfiehlt es sich, Schine r's Worte über Tephritis (Trypeta) eluta M. aus seiner Fauna (II. pag. 171) zu reproduciren: „H. v. Frau enfeld zog. sie aus Centaurea paniculata und Onopordon illyricum; dann in Aegypten aus Amberboa Lippii; die Larve soll aus dem Blüthenkopf in den Stengel und selbst bis zum Wurzelhalse herabsteigen, wo knorrige Stellen ihre Anwesenheit verrathen; nach 
Dufour lebt sie in Centaurea nigra, nach $\mathrm{Kaltenbach} \mathrm{auf}$ Centaurea jacea". - Was nun das Auswandern der Larve aus dem Blüthenkopte in den Stengel betrifft, kann allerdings aus Schiner's Textirung angenommen werden, dass dies auch aut Centaurea und Onopordon geschehe. Auf diesen Pflanzen wurde aber von Niemandem noch eine solche Wanderung bekannt gemacht; die Beobachtung Frau enfeld's aber bezieht sich nur auf Amberboa, so dass also die von Orellia (Tephritis) eluta auf letzterer Pflanze veranlasste Stengeldeformation wohl nicht mit den Aulax-Gallen auf Cent. Scabiosa verglichen werden kann. Fra u enfeld selbst schreibt über die genannte Bohrfliege (in den Sitz. Ber. der k. Acad. d. Wissensch. Wien, Bd. XXII. 1856 , pag. 556) Folg'endes: „Die in Amberboa Lippii und der Flockenblume (Centaurea) lebende, auch in Europa vorkommende und weit verbreitete $T r$. eluta geht gegen die Erfahrungen, die ich aus einer unzähligen Menge von Beispielen ohne eine einzige Ausnahme gewonnen, aus den Blüthenköpfen der Amberboa durch das Anthodium bis an den Stengel, ja ich fand sie auch als Larve und Puppe selbst am Wurzelhalse oder an den Verästelungen des Stammes, wo die Mutterfliege die Eier eingebohrt haben musste, und wo ihre Anwesenheit insoferne leicht bemerkbar wird, als jene Stellen knorrig und aufgetrieben erscheinen."

48. Herr Dr. K. Rech inger (aus Wien) überbrachte mir 2 Exemplare von Trypeta onotrophes Lw., welche ihm aus Köpfchen von Centaurea vochiniensis Bernh. ausgekommen waren. Die Pflanze sammelte Dr. R e chinger im Tarnovaner Walde bei Görz Ende Juli 1894. Die genannte Centaurea war bisher als Futterpflanze von Tr. onotrophes nicht bekannt.

49. Ebenfalls von Herrn Dr. Rechinger erhielt ich mehrere Exemplare von Evaresta (Tephritis) pulchra Lw. mit der Angabe, dass er sie wahrscheinlich aus den Köpfchen von Scorzonera humilis L. aus der Wiener Gegend gezogen habe. Da ich im Frühjahr 1886 diese Fliege nicht selten auf den noch nicht geöffneten Köptchen derselben Pflanze (bei Waldegg in Nied.Oesterreich) gesammelt habe, ist es wahrscheinlich, dass sie im Larvenzustande in der genannten Pflanze lebt. Bisher war nur Podospermum Jacquinianum Koch als Futterpflanze von Evaresta pulchra bekannt. 
50. Herr Dr. C. K e r t é s z beschreibt im Termész. Füzetek, vol. XIX, 1896, pag. 26 eine Pelecocera rectinervis n. sp., welche er in 5 Exemplaren in den Bergen von Buda (Central-Ungarn) im April gesammelt hat. Aus der lateinischen Diagnose und aus der kurzen, ebenfalls lateinischen Discussion über die Verwandtschaft der neuen Art mit Pel. lugubris Perr. (das übrige ist in ungarischer Sprache geschrieben, worüber ich keine weitere Auskunft geben kann) entnimmt man Folgendes: der Hinterleib der neuen Art ist wie bei P. lugubris oberseits einfärbig schwarz; doch ist bei ersterer der Gesichtstheil des Kopfes nicht so weit vorgezogen und sind die Fühler anders gebildet als bei $P$. lugubris, welche beiden Merkmale auch in den beigegebenen Abbildungen ersichtlich gemacht sind. Den Hauptunterschied gegenüber lugubris glaubt der Autor der neuen Art in dem Flügelgeäder erkennen zu müssen, indem er von seiner Art sagt: "nervo longitudinali tertio recto et non in medio sinuato" und sie daher auch rectinervis getauft hat. Dieses Merkmal ist aber s icher ein hinfälliges: es kann sich nur auf das Basalstïck der 3. Längsader (Nomenclatur nach S ch in e r's Fauna) beziehen, welches sich von der Wurzel dieser Ader bis zur kleinen Querader erstreckt. Herr Dr. Kertés z hat sich hierin auf die Abbildung verlassen, welche $\mathrm{P}$ e $\mathrm{r} \mathrm{r}$ is von seiner $P$. lugubris gegeben hat. Wer aber die Gattung Pelecocera näher kennt und wer auch Perris' schematisirte Zeichnungen richtig zu beurtheilen weiss, der wird dem von Perris in der That geschwungen dargestellten Basalstück der genannten Ader keinen specifischen Werth beilegen, umsomehr als P e r r is in der Beschreibung von $P$. lugubris kein Wort hierüber erwähnt. Darauf aber möchte ich noch hinweisen, dass $P$. rectinervis laut der vorliegenden Beschreibung mit Ausnahme der Hinterleibsfärbung vollständig mit $P$. latifrons Lw. übereinstimmt, wemn man nur nicht daran festhält, dass $\mathrm{S}$ ch in e r und L o e w das 3. Fühlerglied "schwarz mit einer gelbrothen Stelle" an der Wurzel beschrieben haben und dass Loew den Bauch gelb nennt. Indem ich mich auf Typen S ch i n e r's, der wieder die Art durch L o e w selbst kennen gelernt hat, berufe, kann ich mittheilen, dass das 3. Fühlerglied schmutzig braungelb ist und gegen oben zu bis in's Schwarze sich verdunkelt, und dass der Bauch eine aschgraue Bestäubung besitzt, unter welcher die Farbe der Hinterleibs-Oberseite gelblich durchscheint. Wenn die Stirn 
des $\sigma^{7}$ von $P$. rectinervis die aussergewöhnliche Breite wie das $\sigma^{T}$ von $P$. latifrons besitzt - Herr Dr. K e rtés z sagt leider in der Diagnose hierüber gar nichts - , so habe ich nicht den geringsten Zweifel, dass wir in $P$. rectinervis einen Melanismus von P. latifrons vor uns haben, wie er ja z. B. bei Catabomba (Syrphus) pyrastri L. nicht selten vorkommt. Die Thatsache, dass Herr Dr. K e r tés z fü n f Exemplare seiner Art - ob an e in er Stelle weiss ich nicht - gesammelt habe, möchte meiner Vermuthung widersprechen, wenn ich nicht selbst an einem und demselben Fangplatze und an einem Tage (in Tirol am Achensee) drei Stücke der melanochroitischen Form von Catab. pyrastri gesammelt hätte.

51. Häufiges Auftreten von Brachycoma devia Fall. Ich habe im verflossenen Sommer bei Hainfeld (in Niederösterreich) sowohl in der Thalsohle als auf den umliegenden Höhen die genannte Tachinine in grosser Menge, man kann sagen nach Hunderten, beobachtet. Die grösste Frequenz zeigte sich in der zweiten Hälfte Julis, während ich die letzten Exemplare noch Ende August getroffen habe. Im Thale waren es die Blüthen von Heracleum Sphondylium L., in der Höhe jene von Laserpitium latifolium L., welche die Fliege mit Vorliebe besuchte. Bekanntlich schmarotzt diese Art in Hummel- und Wespennestern; sie ist nach meinen Beobachtungen $\mathrm{l}$ arvipar und gehört keineswegs zu jenen Arten, welche man als „gemein" zu bezeichnen pflegt.

52. Ueber die Fruchtbarkeit von Stratiomyia chamaeleon Deg. - Ich beobachtete am 2. August des vorigen Jahres ein Weibchen der genannten Fliege, welches Eier ablegte. Die Menge derselben betrug die nicht umbedeutende Zahl von 636! Das Weibchen war eingezwingert, die Eiablage währte fünf Stunden; in der Freiheit wird sich dieses Geschäft wohl schneller abwickeln. Die Eier sind schwefelgelb, glatt, glänzend, sehr dünnschalig, $1.3 \mathrm{~mm}$ lang, $0.2 \mathrm{~mm}$ dick, walzenförmig, gegen die Pole zu etwas verschmälert; sie sind mit einem Klebestoff versehen, mittelst dessen sie locker aneinander halten. Sie traten langsam aus der Legescheide des Weibchens einzeln heraus und bildeten eine Schnur: die meisten berührten sich mit den Polen, manche schoben sich übereinander. Wäre das erstere bei allen Eiern der Fall gewesen, so hätte die Eierschnur die enorme 
Länge von nahezu $1 \mathrm{~m}$ (nämlich $826.8 \mathrm{~mm}$, wenn die Eier als gleich lang angenommen werden) gemessen. - Wenn auch Stratiomyia chamaeleon nicht zu den „selteneren“ Dipteren-Arten gehört, wird man sie kaum je in so grosser Menge antreffen, welche der vorerwähnten Eierzahl entsprechen möchte; mehrere Stratiomyia-Arten, deren Weibchen nicht minder fruchtbar sein dürften, als wir es bei Strat. chamaeleon gesehen haben, sind aber geradezu als sehr seltene Dipteren zu bezeichnen. Der Umstand, dass die Eier und die jungen Larven der Stratiomyien vielfach verschiedenen Wasserthieren zur Beute tallen, ferner, dass diesen Fliegen wahrscheinlich nur ein e Generation zukommt, machen das seltene Auftreten der Imagines trotz der grossen Fruchtbarkeit erklärlich, zugleich aber den Bedarf dieser Fruchtbarkeit, ohne welche die Thiere bei ihrer Lebensweise wahrscheinlich längst schon ausgestorben wären. - Ich habe schon früher einmal von der Fruchtbarkeit eines seltenen Dipterons gesprochen, nämlich in den Verhandl. d. k. k. Zool. Bot. Gesellschft., Jahrg. 1878, pag. 475. Es ist dies Amphipogon spectrum Wahlbg., von welchem ich ein trächtiges Weibchen untersuchen konnte; es trug bei 100 Eier. - Die geringe Fruchtbarkeit von Chirosia trollii Zett. habe ich in der Wien. Entom. Ztg. 1895, pag. 296 constatirt: in dem Abdomen eines Weibchens fand ich nur 10 reife Eier. - Sehr interessant sind die Beobachtungen Abbé K i effer's über die Eierzahl bei Cecidomyiden (siehe den Aufsatz: "Les oeufs des Cécidomyes" in L'Ami d. Sciences natur. Rouen, 1894, Extrait, leider ohne Paginirung!); darnach steigt die Zahl der Eier in einem $\&$ bis gegen 300 (bei Clinorrkiza nigripennis Kieff.), während sie bei p acdogenetischen Formen bis zu fünf herabgeht. Ein weiteres Interesse bietet die geringe Fruchtbarkeit, wie sie von Dr. Portschinsky bei einigen larviparen $A n t h o-$ myinen und M uscinen (1-2 Larven) constatirt wurde (vergl. Hor. Soc. Ent. Ross. XVII. 1882, separ. pag. 1-3). Bekannt ist die geringe Fruchtbarkeit der Hippobosciden.

53. Synonymisches. - Herr Dr. E. Bergroth in Tammerfors theilte mir Folgendes brieflich mit:

"Chrysotoxum fuscum Gigl. T., Atti Acc. Sc. di Torino XXVI, 1890, pag. 160, ist identisch mit Chr. Alavipenne Palma, Sirfidi della fauna Napolit. (Ann. Acc. Asp. Natur. di Napoli, Ser. 3, Vol. III. 1863, sep. pag. 7, tab. I, fig. 1). Dr. Gigilo- 
Tos hat (in litt.) die Richtigkeit dieser Synonymie anerkannt.

Phora interrupta Zett., Ins. lapp. 1840, pag. 797 ist identisch mit der in Schiner's Fauna vergessenen Phora picta Lehm., Zool. Hamb. obs. 1822, pag. 43, tab. I, fig. 6 .

Boletina pseudosciarina Strobl, Mittheil. Nat. Ver. Steierm. Jahrg. 1894 (ausgegeb. 1895), pag. 28 fällt mit B. conformis Siebke, Nyt Mag. f. Nat. 1864, pag. 188 zusammen."

Indem ich dies mittheile, erkläre ich mich mit den Feststellungen Dr. B e rgroth's einverstanden.

54. Ueber Dichocera lyrata Will. - Ich habe ïber diese Tachinine in der Wien. Entom. Ztg. 1895, pag. 103 meine Ansicht bezüglich der Eigenthümlichkeit der Fühlerbildung und bezüglich der systematischen Stellung der Gattung Dichocera ausgesprochen. Ich muss noch einmal darauf zurückkommen und hier zum Verständnisse des Folgenden erwähnen, dass das 3. Fühlerglied beim $\sigma^{\pi}$ von D. Iyrata in zwei Theile tief gespalten ist, wonach Williston die Gattung auch benannte, während es bei dem o einfach, oder höchstens an der Basis mit einem kleinen Vorsprunge versehen ist, ferner, dass Wi llis t o n die Gattung Dichocera in die Verwandtschaft von Nemoraea (sensu Schin.) bringt. Während ich nun l. c. erklärte, dass die Spaltung des 3. Fühlergliedes bei Dichocera $\left(\sigma^{\top}\right)$ wahrscheinlich eine normale Bildung sei (sicher erschien mir dies aber nicht, weil ja das 3. Fühlerglied beim \& nach den Angaben Willis to n's variabel ist), und während ich die Gatt. Dichocera in die Gruppe der Thryptoceratinen versetzen zu dürfen glaubte, sah sich Prof. Dr. Bra u er (in den Sitz. Ber. d. k. Acad. d. Wissensch. Wien, 1895, pag. 601) veranlasst, meiner Ansicht bezüglich der Fühlerbildung wohl beizupflichten, hingegen in Bezug auf die systematische Stellung der Gattung Williston rechtzugeben. In Bezug auf die Fühlerbildung von Dichocera hat aber Prof. B r a u e r, wenn er l. c. sagt: „es wurden (von Williston) $4 \sigma^{7}$ und 15 o mit gleichem Fühlerbau untersucht" übersehen, dass Will i s t on (in Entom. News 1895, pag. 32 ) ausdrïcklich schreibt: „the 3. joint (of the antennae) ... is obtusely pointed at the tip; in some specimens the 3. antennal joint shows a slight projection near the proximal end in front, as though corresponding to a rudiment of the elongated process of the male". -- Was die systematische Stellung. von Dichocera Will. betrifft, schreibt B r a u e r (l. c.): „Dicho- 
cera kenne ich nicht in natura, möchte aber die Gattung $\mathrm{n}$ a $\mathrm{ch}$ dem Profil mit vortretendem Mundrande und dem ganzen Kopfbau, den haarigen Augen, erweiterten Vordertarsen des $q$ etc. in die Nähe von Erigone stellen. Williston sieht sie als nächste Verwandte zu Nemoraea an, in welche Gattung ja früher alle Erigonen gehörten. M i k (Wien. Ent. Ztg. 1895) will sie muthmasslich zur Gruppe Thryptocera als Abnormitäten (!) reihen, wofür zwar die Orbitalborsten des o und o und deren kleine Klauen sprechen, nicht aber das Profil und das Geäder." - Prof. B r a u r kann mich durch diese Auseinandersetzungen von meiner in der Wien. Entom. Ztg. 1895, pag. 601 begründeten Ansicht nicht abbringen, obzwar ich die Typen von Dichocera nicht kenne. Zum mindesten möchte ich Dichocera nicht in die Verwandtschaft mit Erigone bringen; denn dann wäre Brauer's Section Erigone (Verhandl. k. k. Zool.-Botan. Gesellsch. Wien, 1893) unzureichend charakterisirt und verlöre das Gleichgewicht vollständig. Ich kenne keine Art und somit auch keine Gattung der Gruppe Erigone, bei welcher das zweite Fühlerglied die relative Kürze und das dritte die enorme Länge hätte, wie sie Williston von Dichocera abbildet und beschreibt; ebensowenig hat eine Erigone bis zur untersten Augenecke herabsteigende Borsten (als Fortsetzung der Stirnborsten). Abgesehen von den Orbitalborsten und kurzen Klauen (sowie Pulvillen) bei beiden Geschlechtern von Dichocera, was ja bei Erigone nicht vorkommt, ist überdies der Mundrand bei letzterer Gattung viel mehr vorspringend als die Abbildung von Dichocera zeigt und somit das Profil des Kopfes und dessen Bildung, auf welche Merkmale sich Br a ue r besonders stützt, ein sehr verschiedenes. - Soweit aus der Abbildung und Beschreibung von Dichocera zu entnehmen ist, sind viel mehr Chancen vorhanden, dieselbe zur Section Thryptocera zu stellen. Der Kopf gleicht, was die relative Länge der Fühlerglieder und die Bildung der Fïhlerborste, ferner was das P r o fil betrifft, wohl gar sehr einer Admontia (conf. Brau. Berg. Muscaria schizometopa P. I. Tab. V, Fig. 92); die Beborstung der Wangen findet sich bei gewissen Thryptoceren wie bei Dichocera, ebenso kommen behaarte Augen vor; Admontia hat im weiblichen Geschlechte die Vordertarsen erweitert. Williston selbst schreibt von Dichocera: „first posterior cell narrow and narrowly open, the apical cross-vein oblique, terminating a little distance before 
the tip of the wing; . . . angle with a stump of a vein". Dies alles finden wir bei Ptychoneura (B. B. l. c. Tab. V, Fig. 96), welche gleichfalls zu den Thryptoceratinen gehört. Dazu kommt noch, wie erwähnt, das für diese Gruppe essentielle Merkmal der Orbitalborsten und kurzen Klauen bei beiden Geschlechtern. - Immerhin aber gebe ich gerne zu, dass ohne Kenntniss der Typen von Dichocera meine Auffassung bezüglich ihrer Stellung bei den Thryptoceratinen eine irrthümliche sein möge; ich wiederhole aber, dass man noch weniger berechtigt ist, die NemoraeaGruppe (im Sinne der älteren Autoren) als Verwandtschaftskreis von Dichocera anzusehen. Weit mehr für sich hätte die alte Gattung Exorista, in welche Dichocera lyrata besser passen würde als zu Nemoraea. Ich möchte dann Exorista ruficauda Zett. als eine ziemlich nahe Verwandte von Dichocera ansprechen, obwohl ihr die Orbitalborsten im männlichen Geschlechte fehlen; sie hat in beiden Geschlechtern kurze Krallen, das o besitzt verbreiterte Vordertarsen, die Stirnborsten zeigen die Tendenz weit herabzusteigen und die relative Länge der Fühlerglieder entspricht mehr der Gattung Dichocera als es bei Erigone (Nemoraea) der Fall ist. Bekanntlich hat B r a u e r auf die genannte Exorista die Gattung Micronychia begründet und dieselbe in seine Sectio Polidea gestellt. Hier könnte also auch Dichocera gesucht werden, wenn sie nicht zu den Thryptoceratinen gehören sollte. - B r a u e gebührt das Verdienst, aus dem ungeheuern Wust der Tachiniden-Literatur noch auf zwei Arten mit gespaltenem dritten Fühlerglied hingewiesen zu haben (Sitz. Ber. l. c. pag. 600). Es sind dies Tachina convecta und exul, welche Wa lke r (Insecta Saund. 1856, pag. 276 - 277) aus den Vereinigten Staaten Nord-Amerika's beschrieben und in die Gatt. Schizotachina sibi (l. c. pag. 264) gestellt hat. Nach der Beschreibung der Fühlerborste gehören meines Erachtens beide Arten in verschiedene Gattungen. - Herr Van der Wulp beschrieb in neuester Zeit noch eine Tachinine mit gespaltenem dritten Fühlergliede aus Java. Er errichtet auf die Art die neue Gattung Diglossocera (Tijdschr. v. Entomologie, 1895, pag 51) und spricht in einem (lückenhaften) Nachtrage zu diesem Artikel (l. c. pag. 163) über die bekannten Arten mit ähnlicher Fühlerbildung. In keiner von diesen Abhandlungen berührt er meinen Artikel (Wien. Entom. Ztg. 1895, pag. 101) über den gleichen Gegenstand; es mag immerhin sein, dass ihm derselbe noch nicht zur Hand sein konnte, als er seinen "Nachtrag“" schrieb. 


\section{$2 \mathrm{BHL}$ Biodiversity Heritage Library}

Mik, Josef. 1896. "Dipterologische Miscellen. (2. Serie)." Wiener entomologische Zeitung 15, 106-114. https://doi.org/10.5962/bhl.part.13868.

View This Item Online: https://www.biodiversitylibrary.org/item/43774

DOI: https://doi.org/10.5962/bhl.part.13868

Permalink: https://www.biodiversitylibrary.org/partpdf/13868

\section{Holding Institution}

Smithsonian Libraries

\section{Sponsored by}

Smithsonian

\section{Copyright \& Reuse}

Copyright Status: NOT_IN_COPYRIGHT

This document was created from content at the Biodiversity Heritage Library, the world's largest open access digital library for biodiversity literature and archives. Visit BHL at https://www.biodiversitylibrary.org. 